\title{
Elemental principles of t-topos
}

\author{
G. KATO
}

PACS. 03.65.Ud - Entanglement and quantum nonlocality (e.g. EPR paradox, Bell's inequalities, GHZ states, etc.).

PACS. 03.65.Ta - Foundations of quantum mechanics; measurement theory.

PACS. 04.20. Cv - Fundamental problems and general formalism.

\begin{abstract}
In this paper, a sheaf-theoretic approach toward fundamental problems in quantum physics is made. For example, the particle-wave duality depends upon whether or not a presheaf is evaluated at a specified object. The $t$-topos theoretic interpretations of doubleslit interference, uncertainty principle(s), and the EPR-type non-locality are given. As will be explained, there are more than one type of uncertainty principle: the absolute uncertainty principle coming from the direct limit object corresponding to the refinements of coverings, the uncertainty coming from a micromorphism of shortest observable states, and the uncertainty of the observation image. A sheaf theoretic approach for quantum gravity has been made by Isham-Butterfield in (Found. Phys., 30 (2000) 1707), and by Raptis based on abstract differential geometry in Mallios A. and Raptis I., Int. J. Theor. Phys., 41 (2002), qr-qc/0110033; Mallios A., Remarks on "singularities" (2002) qr-qc/0202028; Mallios A. and Raptis I., Int. J. Theor. Phys., 42 (2003) 1479, qr-qc/0209048. See also the preprint by REQuARDT M., The translocal depth-structure of space-time, Connes' "Points, Speaking to Each Other", and the (complex) structure of quantum theory, for another approach relevant to ours. Special axioms of t-topos formulation are: i) the usual linear-time concept is interpreted as the image of the presheaf (associated with time) evaluated at an object of a $t$-site (i.e., a category with a Grothendieck topology). And an object of this t-site, which is said to be a generalized time period, may be regarded as a hidden variable and ii) every object (in a particle ur-state) of microcosm (or of macrocosm) is regarded as the microcosm (or macrocosm) component of a product category for a presheaf evaluated at an object in the $t$-site. The fundamental category $\hat{S}$ is defined as the category of $\prod_{\alpha \in \Delta} C_{\alpha}$-valued presheaves on the $t$-site $S$, where $\Delta$ is an index set. The study of topological properties of $S$ with respect to the nature of multi-valued presheaves is left for future study on the t-topos version of relativity (see Kato G., On t.g. Principles of relativistic t-topos, in preparation; Guts A. K. and GRINKEvich E. B., Toposes in General Theory of Relativity (1996), arXiv:gr-qc/9610073, 31). We let $C_{1}$ and $C_{2}$ be microcosm and macrocosm discrete categories, respectively, in what will follow. For further development see also Kato G., Presheafification of Matter, Space and Time, International Workshop on Topos and Theoretical Physics, July 2003, Imperial College (invited talk, 2003).
\end{abstract}


Basic definitions. - For t-topos theory, the notion of a t-site plays the role of hidden variables. More conditions will be added to the site when the further applications in [1] are made. For the concept of a Grothendieck topology, see [2-4] or [5].

Definition 1.1. Let $S$ be a site, namely, a category with a Grothendieck topology and let $\hat{S}$ be the category of presheaves from $S$ to the product category $\prod_{\alpha \in \Delta} C_{\alpha}$. That is, $\hat{S}=\left(\prod_{\alpha \in \Delta} C_{\alpha}\right)^{S^{\text {opp }}}$, where $S^{\text {opp }}$ is the dual category of $S$. Then site $S$ is said to be a temporal site or simply $t$-site when $S$ is used in this context. Category $\hat{S}$ is said to be a t-topos or temporal topos. We sometimes call an object of $\hat{S}$ an entity.

Remarks 1.2. i) See [3] or [5] for Grothendieck topologies which is sufficient for t-topos theory.

ii) For an object $F$ in $\hat{S}$, which we write as $F \in O b(\hat{S})$ and for an object $V$ in $S$, i.e., $V \in O b(S), F(V)$ is an object in $\prod_{\alpha \in \Delta} C_{\alpha}$. Namely,

$$
F(V)=\left(F(V)_{\alpha}\right)_{\alpha \in \Delta}
$$

where $F(V)_{\alpha}$ is the $\alpha$-th component of $F(V)$. We also say that $F(V)$ is the manifestation of $F$ at the generalized time period $V$.

Definition 1.3. Let $F$ be an object of $\hat{S}$. The state of $F$ during a generalized time period $W$, namely, an object of $S$, is defined by the pair $(F, W)=F(W)$. Then $F$ is said to be manifested during $W$. When a generalized time period is not given, $F$ is said to be in a pre-state or in an unmanifested state. (See Note $1.4^{\prime}$ below.) For a specified object $V$, the object $F(V)$ is said to be in the particle ur-state of $F$ over the generalized time period $V$, and when one object in the t-site is not specified for $F$, then $F$ is said to be in a wave ur-state of $F$ and sometimes denoted as $\{F(W)\}_{W \in O b(S)}$ or $F(-)$.

Definition 1.4. An observation of an object $m$ of $\hat{S}$ by another object $P$ of $\hat{S}$ in a nondiscrete category $C_{\alpha}, \alpha \in \Delta$, over a generalized time period $V$ is a natural transformation $s$ over $V$. Namely, the morphism in $C_{\alpha}$

$$
s_{V}: m(V) \longrightarrow P(V)
$$

is said to be an observation of $m$ by $P$ during the generalized time period $V$. If such a natural transformation $s$ over a specified object $V$ of t-site exists, then $m$ is said to be observable or measurable by $P$ during the generalized time period $V$. We may also say that $m$ interacts with $P$ if there exists such a natural transformation from $m$ to $P$ over some generalized time period. Notice that when $m$ is measured, $m$ needs to be in a particle ur-state since an object in $S$ must be specified for the natural transformation in (1).

Note $1.4^{\prime}$. When an object $m$ of $\hat{S}$ is not observed, not only $m$ is in the wave ur-state, i.e., $\{m(V)\}$ in Definition 1.3, but also (we will be more precise in Definitions 2.1 and 2.2) $m$ may be considered as the totality of decomposed subobjects of $m$ which are to be evaluated at unspecified objects of $S$. It may be most appropriate to consider an unobserved object $m$ to be simply presheaf " $m$ ".

Note 1.5. Let $\left\{V_{i} \rightarrow V\right\}$ be a covering of $V$ and let $\left\{V_{i \leftarrow j} \rightarrow V_{i}\right\}$ be a covering of $V_{i}$ as in [2-6] or [7]. Then by composing covering morphisms, $\left\{V_{i \leftarrow j} \rightarrow V\right\}$ is a covering of $V$. Similarly, by composing further, one gets a covering $\left\{V_{k \leftarrow j \leftarrow i} \rightarrow V\right\}$ of $V$. Then, consider the inverse limit covering

$$
\left\{\lim _{\leftarrow} V \ldots \leftarrow k \leftarrow j \leftarrow i \longrightarrow V\right\}
$$

of $V$. In the next section, we will need this notion. 
Definition 1.6. Let $C_{1}$ be the microcosm discrete category. That is, an object of $C_{1}$ is a particle in microcosm, and as a category, $C_{1}$ is discrete, namely, no morphisms exist except identity morphisms.

Note 1.7. The topos approach in [8] and [9] by Butterfield-Isham can be interpreted in terms of t-topos as follows. First, we will explain the basic method in [8] and [9]: Let $S$ be the state space and let $A$ be a physical quantity and let $\bar{A}$ be a real-valued function representing $A$ as in [8]. Then the functional composition principle (referred to as FUNC in [8] and [9]) is the commutative diagram

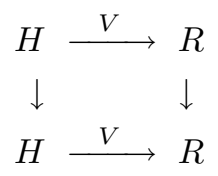

where the left-hand side vertical morphism $\tilde{h}: H \rightarrow H$ on the Hilbert space $H$ is induced by a function $h: R \rightarrow R$ on real numbers. That is, for the value $V(\hat{A})$ of the physical quantity $A$ represented by the operator $\hat{A}$, we have $V(\tilde{h}(\hat{A}))=h(V(\hat{A}))$ which is the commutativity of the above diagram. The Butterfield-Isham topos theory interprets this commutative diagram as follows: Regarding the valuation $V$ as a natural transformation $\gamma$ from a terminal object 1 to an object $X$ in the topos of presheaves, for $\hat{f}: \hat{B} \rightarrow \hat{A}$ in the category of all bounded self-adjoint operators, we first have $X(f): X(A) \rightarrow X(B)$, and $\gamma_{A}$ in $X(A)$ and $\gamma_{B}$ in $X(B)$, since $\gamma$ is a natural transformation from 1 to $X$. Here we make the following interpretation of $\gamma_{A}$ as a morphism from $A$ to $X$ using Yoneda Lemma. Then the Kochen-Specker Theorem states that such a global section $\gamma$ does not exist to satisfy the commutative diagram

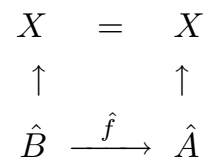

where the vertical morphisms are $\gamma_{A}$ and $\gamma_{B}$. Namely, $\gamma_{B}=\gamma_{A} \circ \hat{f}$, i.e., $X(f)\left(\gamma_{A}\right)=\gamma_{B}$, the matching condition in $[8,9]$. In terms of t-topos, the value $V^{s}(A)$ of $A$ at a state $s \in S$ corresponds to $m(V)$ over $V \in O b(S)$, where $m \in O b(\hat{S})$. Suppose that usual linear time $\tau(V)$ precedes $\tau(U)$. And let $g: V \rightarrow U$ be the associated morphism in the t-site $S$. (See [10] for the associated morphism induced by the linear ordering on $\tau$.) Then, the t-topos version of Kochen-Specker Theorem states that there does not exist a natural transformation s over the t-site $S$ (itself) making the diagram

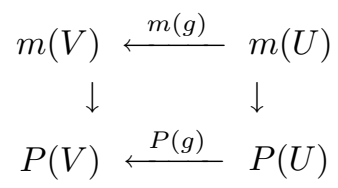

commutative, where the left-hand side vertical morphism is $s_{V}$ and the right-hand side vertical morphism is $s_{U}$ as in Definition 1.4. Note that such a globally defined natural transformation $s$ (which is $\gamma$ in Butterfield-Isham) from $m$ to $P$ is defined for the entire objects of $S$ (a global section from 1 to $X)$. As for t-topos, the definition of an observation is defined for a specified object of $S$ as in Definition 1.4.

Note 1.8. Every object in $C_{1}$ is the $C_{1}$-component of a presheaf in $\hat{S}$ evaluated at a generalized time period in the t-site $S$. For example, if $\underline{\text { e }}$ is a particle in $C_{1}$, then there exists 
an associated presheaf $e$ in $\hat{S}$ such that for an object $V$ in the t-site $S$, we have e $=e(V)$, which

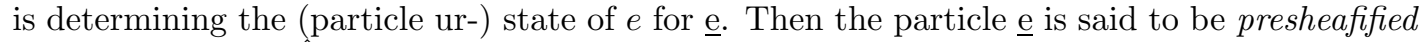
by a presheaf $e$ in $\hat{S}$.

Remark 1.9. Let $\mathrm{e}$ be any object in $C_{1}$. For example, e can be an electron. For a particle e in $C_{1}$, the position and time $x$ and $t$ are associated locally. As is mentioned in Note 1.8, we presheafify $\underline{\mathrm{e}}$ as $\underline{\mathrm{e}}=e(V)$ in $C_{1}$, where $e \in O b(\hat{S})$ and $V \in O b(S)$. We will presheafify the position by presheaf $\kappa$ and time by presheaf $\tau$ defined over the same object $V$ in the third section, so that $(\kappa(V), \tau(V))$ plays a local coordinate system of $e(V)$.

Definition 1.10. Let $m_{1}, m_{2}, \ldots, m_{r}$ be objects of $\hat{S}$. If the $r$-tuple $\left(m_{1}, m_{2}, \ldots, m_{r}\right)$ can be considered as one object of $\hat{S}$ over a subsite, then objects $m_{1}, m_{2}, \ldots, m_{r}$ are said to be ur-entangled (or ur-correlated). See [11] for the application to the EPR-type non-locality.

Sheaf-theoretic methods for non-locality and sub-Planck region. - Let $m$ and $m^{\prime}$ be the presheaves associated with $\underline{\mathrm{m}}$ and $\underline{\mathrm{m}}^{\prime}$ and let $(\kappa, \tau)$ be the associated sheaves to space and time to $m$. Suppose that $m$ and $m^{\prime}$ are ur-entangled as defined in Definition 1.10. Furthermore, assume that $m(V)$ and $m^{\prime}(V)$ are physically a distance apart (for the same object $V$ ), e.g., 11 kilometers apart. Then the space-time $(\kappa(V), \tau(V))$ of $m(V)$ does not contain $m^{\prime}(V)$, if necessary by taking $V$ "small" enough in the sense of a covering. That is, space-time presheaves are associated with $m$ in $\hat{S}$. Namely, $(\kappa, \tau)$ should be denoted as $\left(\kappa_{m}, \tau_{m}\right)$, see also [11].

Definition 2.1. Let $M$ be a matter in the macrocosm discrete category $C_{2}$. Let $m$ be the associated presheaf to $M$. Then a finite direct sum of presheaves $\sum_{\lambda \in \Lambda} m_{\lambda}$ of $m$ is said to be a uniform quantum decomposition of $m$ with respect to a covering $\left\{V_{\lambda} \rightarrow V\right\}$ of a generalized time period $V$ if each $m_{\lambda}$ is an object of $\hat{S}$ so that $m_{\lambda}\left(V_{\lambda}\right)$ may be an object of $C_{1}$, and $\sum_{\lambda \in \Lambda} m_{\lambda}\left(V_{\lambda}\right)=m(V)$. See [10] for the notion of a sub-Planck decomposition.

Remark 2.2. A short remark on Double-Slit Interference may be appropriate, see [12] for details. Suppose that an electron $\underline{e}$ is fired at a certain time. In terms of t-topos, $\underline{\mathrm{e}}=e(V)$ is fired at $(\kappa(V), \tau(V))$, where $e, \kappa$, and $\tau$ are associated presheaves to the electron $\underline{\mathrm{e}}$, space and time. Assume also that two slits are appropriately narrow and the spacing between the slits is much larger than the width of the slit. Let $(\kappa(U), \tau(U))$ be the position and the time when the electron hits the screen, inducing a morphism $g: V \rightarrow U$. For the two slits, let $W$ and $W^{\prime}$ be the associated objects of the t-site $S$ for which $(\kappa(W), \tau(W))$ and $\left(\kappa\left(W^{\prime}\right), \tau\left(W^{\prime}\right)\right)$ would be the corresponding slits that $\mathrm{e}$ would go through. Without an observation at either one of the slits, there are two objects, i.e., $W$ and $W^{\prime}$, in $S$. Hence, by Definition 1.3, e remains to be in a wave ur-state. In the case where there is no mask between the screen and an electron gun, one needs to consider not only via $W$ and $W^{\prime}$, but also all the factorizations of $g: V \rightarrow U$. Then $e$ is in the wave ur-state $e(\{g: V \rightarrow U\})$, where $\{g: V \rightarrow U\}=\{W \in O b(S): g=f \circ h$, where $f: V \rightarrow W$ and $h: W \rightarrow U\}$, see [12] for details.

Remark 2.3. One can choose a covering $\left\{V_{i} \rightarrow V\right\}$ and another covering $\left\{V_{i \leftarrow j} \rightarrow V\right\}$ as in Note 1.5 , so that $m\left(V_{i}\right)$ and $m\left(V_{i \leftarrow j}\right)$ may belong to $C_{1}$ and the Planck scale category $C_{\mathrm{Pl}}$, respectively.

Remark 2.4. First note, for example, when we consider the $C_{1}$-components of $m(V)$ and $P(V)$ in Definition 1.4, such a morphism as $s_{V}$ in (1) belongs to a non-discrete category $C_{\alpha}$. However, in the following, we simply say that $s_{V}$ is an observation of $m(V)$ by $P(V)$ in $C_{1}$. An $\hat{S}$-theoretic interpretation of an observation of an electron by an observer is the following. Let $e$ be the presheaf in $\hat{S}$ corresponding to an electron e. Let $P$ be an observer, i.e., an object of $\hat{S}$ and let $V$ be an generalized time period. As defined in Definition 1.4, an observation of $e$ by $P$ is a natural transformation $s_{V}$ from $e$ to $P$ over $V \in O b(S)$. 
Remark 2.5 (Uncertainty principles). Suppose time $\tau(V)$ precedes $\tau(U)$ inducing a morphism $V \stackrel{\varepsilon}{\rightarrow} U$ as in Note 1.7. We define the notion of a micromorphism as follows: the morphism $V \stackrel{\varepsilon}{\rightarrow} U$ is said to be a micromorphism if the morphism $\varepsilon$ can not be factored as $\varepsilon=\beta \circ \alpha$, where $\alpha: V \rightarrow W$ and $\beta: W \rightarrow U$ and so that $\tau(V)$ may precede $\tau(W)$ which precedes $\tau(U)$. For a general morphism $g: V \rightarrow U$, one can consider a micro-decomposition of $g$ as follows: $g=g_{n} \circ \ldots \circ g_{0}$ and each $g_{j}: V_{j-1} \rightarrow V_{j}$ is a micromorphism. A consequence of a micromorphism $V \stackrel{\varepsilon}{\rightarrow} U$ is that it is impossible to observe a particle (presheaf) between $\tau(V)$ and $\tau(U)$ by the definition. Consequently, the position of particle $m$ between $\tau(V)$ and $\tau(U)$ cannot be known (observed). Since $\kappa$ and $\tau$ are ur-entangled, we also obtain the uncertainty in position as well. (See the following third section and [10], and [4] for the relativistic version.) The above uncertainty corresponds to the usual Heisenberg uncertainty principle in the following sense. For a micromorphism $V \stackrel{\varepsilon}{\rightarrow} U$, the difference in position $\kappa(U)-\kappa(V)$ times the difference in momentum $p(U)-p(V)$ is not less than $\hbar$, where $p$ is the presheaf associated with momentum.

There is another uncertainty principle that is absolute in nature. Since we have replaced the notion of a set theoretic point with the notion of an object of a category, we have a finite value of the direct limit over coverings $\lim \tau\left(V_{\ldots \leftarrow j \leftarrow i}\right)$. (Note that it is not the inverse limit since a presheaf is contravariant.) Similarly, we have the absolute uncertainty for position sheaf. This material is expected to be expanded in a forthcoming paper [1].

Remark 2.6 (Definition 1.10 and the EPR). Let $\underline{\mathrm{e}}$ and $\underline{\mathrm{e}}^{\prime}$ be entangled electrons, and let $e$ and $e^{\prime}$ be the associated presheaves which are ur-entangled. Namely, the pair $\left(e, e^{\prime}\right)$ is an object of $\hat{S}$ satisfying $\underline{\mathrm{e}}=e(V)$ and $\underline{\mathrm{e}}^{\prime}=e^{\prime}(V)$ for the common object $V$ in a subsite as in Definition 1.10. Then $e^{*}=\left(e, e^{\prime}\right) \in O b(\hat{S})$. For a specified generalized time period $V$, we have objects $e(V)$ and $e^{\prime}(V)$. That is, the states of $\underline{\mathrm{e}}$ and $\underline{\mathrm{e}}^{\prime}$ are determined by the generalized time period $V$ and are independent of the physical distance between $e(V)$ and $e^{\prime}(V)$ in $C_{2}$. When $e$ is observed or measured by $P$ in the sense of Definition 1.4, there is a morphism $s_{V}: e(V) \rightarrow P(V)$ for some $V$ in $S$. This $V$ determining the state of the object $e$ simultaneously determines the state of $e^{\prime}$ in the sense of Definition 1.10, see [11] for the full-length description of this topic.

\section{Sheafification of space and time.}

Axiom 3.1 (Interpretation of the physical time as a sheaf). As in Remark 1.9, we have already noted that the physical time depends upon generalized time. That is, we hypothesize that $\tau$ is an object of $\hat{S}$ so that $\tau(V)$ is the (local) physical time. Then by this definition of the usual physical time, time is of local nature in the sense that for any object $V$ of t-site $S, \tau(V)$ may exist only locally and may not be globally extended. (See the first paragraph of the second section.) For the dependency of $\tau$ on a (ur-)particle is a consequence of (ur-)entanglement as we noted earlier.

Axiom 3.2 (Interpretation of the physical space as a sheaf). Let $\kappa$ be the sheaf associated with the physical space with dimension $d$. That is, for an object $V$ of $S, \kappa(V)$ is the local physical space in $C_{1}$ (or in $C_{2}$ ) of dimension $d$. Then decompose $\kappa(V)$ as $\kappa(V)=\left(\kappa(V)^{3}, \kappa(V)^{d-3}\right.$ ) so that $\kappa(V)^{3}$ may be the observable object of $C_{1}$, and $\kappa(V)^{d-3}$ may be non-observable in $C_{1}$.

Note 3.3. A motivation for Axioms 3.1 and 3.2 is the following. The objects $\kappa$ and $\tau$ in $\hat{S}$ are not only presheaves but also need to be sheaves so that the discrete concept of (pre)sheaves can give the continuum notion of space-time in macrocosm when local data agree on overlaps as in the definition of a sheaf, see [10] a for more thorough treatment of sheaves $\kappa$ and $\tau$. In [1], as an application to quantum gravity, the following case is considered: let $m, m^{\prime}$, 
$P, P^{\prime} \kappa$ and $\tau$ be in $\hat{S}$ and let $V \stackrel{\varepsilon}{\rightarrow} U$ be a micromorphism in $S$, where $m(V)$ and $m^{\prime}(V)$ have non-zero mass and the intersection of $\kappa(V)$ and $\kappa(U)$ is not empty. Then the commutative diagram is induced from $V \stackrel{\varepsilon}{\rightarrow} U$ and morphisms among $m, m^{\prime}, P$, and $P^{\prime}$. When $m(V)$ is massless, the morphism from $P(V)$ to $P^{\prime}(V)$ becomes a Lorentz morphism.

Conclusion. - With our model in terms of t-topos $\hat{S}$, particles, space and time are presheafified, and then the interplay among the concepts of observation, wave-particle urstates, uncertainty principles, non-locality (entanglement), and quantum fluctuation are phrased in terms of objects, morphisms, and sheaves. The sequence of dependency is the following:

$$
\begin{gathered}
\hat{S} \stackrel{\text { evaluated at } O b(S)}{\longrightarrow} \prod_{\alpha \in \Delta} C_{\alpha} \stackrel{\text { projection }}{\longrightarrow} C_{1}\left(\text { or } C_{2}\right) . \\
\\
* * *
\end{gathered}
$$

The author is thankful for the referee's suggestions including I) to make some connections to the topos theory of [8] and [9], and II) to include some applications of t-topos, and III) to make connections to quantum gravity.

\section{REFERENCES}

[1] Kato G., On t.g. Principles of relativistic t-topos, in preparation.

[2] Artin M., Grothendieck Topology, Mimeographed Notes (Harvard University) 1962.

[3] Gelfand S. I. and Manin Yu. I., Methods of Homological Algebra (Springer) 1996.

[4] Kato G., Kohomolojii No Kokoro (Iwanami-Shoten, Tokyo) 2003 (in Japanese).

[5] Kato G., The Heart of Cohomology, to be published by Kluwer-Springer (2005).

[6] Kato G. and Struppa D. C., Fundamentals of Algebraic Microlocal Analysis (Marcel Dekker, Inc.) 1999.

[7] Shubert H., Categories (Springer-Verlag, New York, Heidelberg Berlin) 1972.

[8] Butterfield J. and Isham C. J., Int. J. Theor. Phys., 37 (1998) 2669, quantum ph/980355.

[9] Butterfield J. and Isham C. J., Int. J. Theor. Phys., 38 (1999) 827, quantum ph/9808067.

[10] Kato G., Presheafification of Matter, Space and Time, in Proceedings of International Workshop on Topos and Theoretical Physics, July 2003, Imperial College (invited talk) preprint (2004).

[11] Kafatos M., Kato G. and Roy S., Sheaf cohomology and geometrical approach to EPR nonlocality, to be submitted to Int. J. Pure Appl. Math.

[12] Kato G. and Tanaka T., Double-Slit Interference and t-Topos, to be submitted to Found. Phys. 